\title{
Invasive Pneumococcal Disease Associated with Fanconi-Like Syndrome
}

\author{
Jade Xiao Jue Soh, Raymond Kai Heng Goh, Shuwei Zheng \\ Department of General Medicine, Sengkang General Hospital, Singapore
}

Received: 24/07/2019

Accepted: $12 / 08 / 2019$

Published: $27 / 09 / 2019$

How to cite this article: Soh JXJ, Goh RKH, Zheng S. Invasive pneumococcal disease associated with Fanconi-like syndrome. EJCRIM 2019;6: doi:10.12890/2019_001230.

Conflicts of Interests: The Authors declare that there are no competing interest

This article is licensed under a Commons Attribution Non-Commercial 4.0 License

\section{ABSTRACT}

Acquired causes of Fanconi syndrome in adults are usually due to drugs, toxins or paraproteinaemias. Infectious causes are rarely described. We report a case of invasive pneumococcal disease in a patient who developed a Fanconi-like syndrome during the course of her illness. This patient presented with multiple electrolyte derangements consisting predominantly of hypokalaemia, hypomagnesaemia and hypophosphataemia during hospitalization for invasive pneumococcal disease with possible Austrian syndrome. Further evaluation revealed significant urinary losses of these electrolytes, uric acid and $\beta 2$-microglobulin. Together with evidence of hypouricaemia, this is suggestive of proximal renal tubulopathy, and hence a Fanconi-like syndrome. The patient's clinical condition and biochemical anomalies improved following pneumococcus treatment.

\section{LEARNING POINTS}

- Suspect Fanconi syndrome when there are multiple electrolyte derangements consisting of hypokalaemia, hypomagnesaemia and hypophosphataemia.

- Recognise the common causes of Fanconi syndrome and appreciate that infections such as legionellosis, leptospirosis and pneumococcal disease can potentially result in Fanconi syndrome.

- The management of Fanconi syndrome is generally supportive and involves treating the underlying cause.

\section{KEYWORDS}

Pneumococcus, Streptococcus pneumoniae, Fanconi syndrome, proximal renal tubulopathy

\section{CASE DESCRIPTION}

An 83-year-old woman presented with cough, fever and delirium secondary to invasive pneumococcal disease (IPD) and possible Austrian syndrome. Blood cultures grew Streptococcus pneumoniae. A lumbar puncture performed following antimicrobial therapy showed cerebrospinal fluid pleocytosis, raised protein and pneumococcal antigen (Alere BinaxNOW ${ }^{\circledR}$ ) positivity. Her chest radiograph (Fig. 1) on admission revealed left lung consolidation. Multiple cortical and subcortical infarcts were seen on magnetic resonance imaging of her brain (Fig. 2), suggesting a cardioembolic source. Dysmorphic red blood cells were seen on urine phase contrast. Transthoracic echocardiography was negative for vegetation and she was too unwell to undergo transoesophageal echocardiography.

The patient's medical history included cervical cancer which had been treated with radiotherapy 3 years ago, without recurrence. Apart from simvastatin for dyslipidaemia, the patient was not taking any other medications, including over-the-counter or traditional medicines. Her previous serum electrolytes during routine follow-up were normal and there had been no hospitalization during the previous 3 years. 


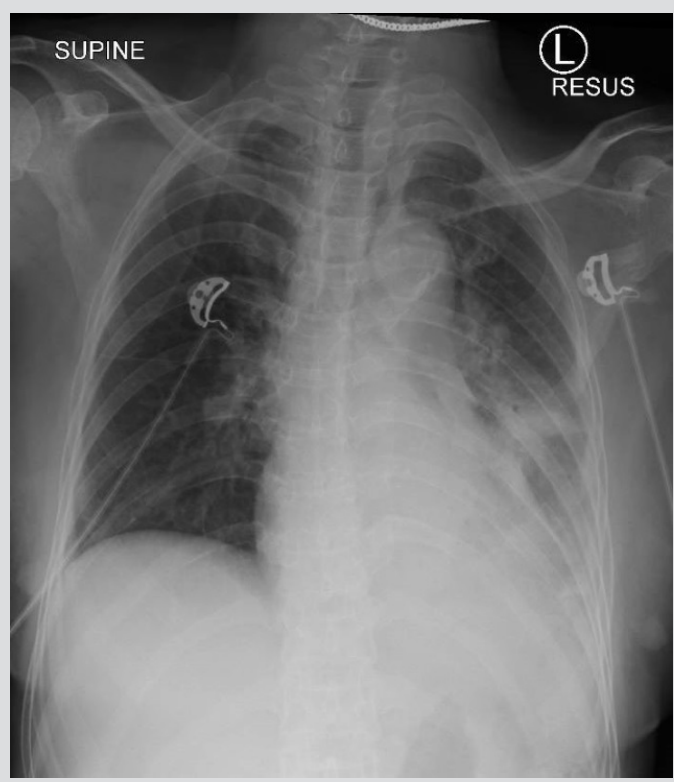

Figure 1. Patchy air-space opacities in the left mid and lower zones on chest radiograph Figure 2. Scattered foci of restriction diffusion (white arrows) in bilateral cortical, subcortical and deep white matter regions on magnetic resonance imaging of the brain
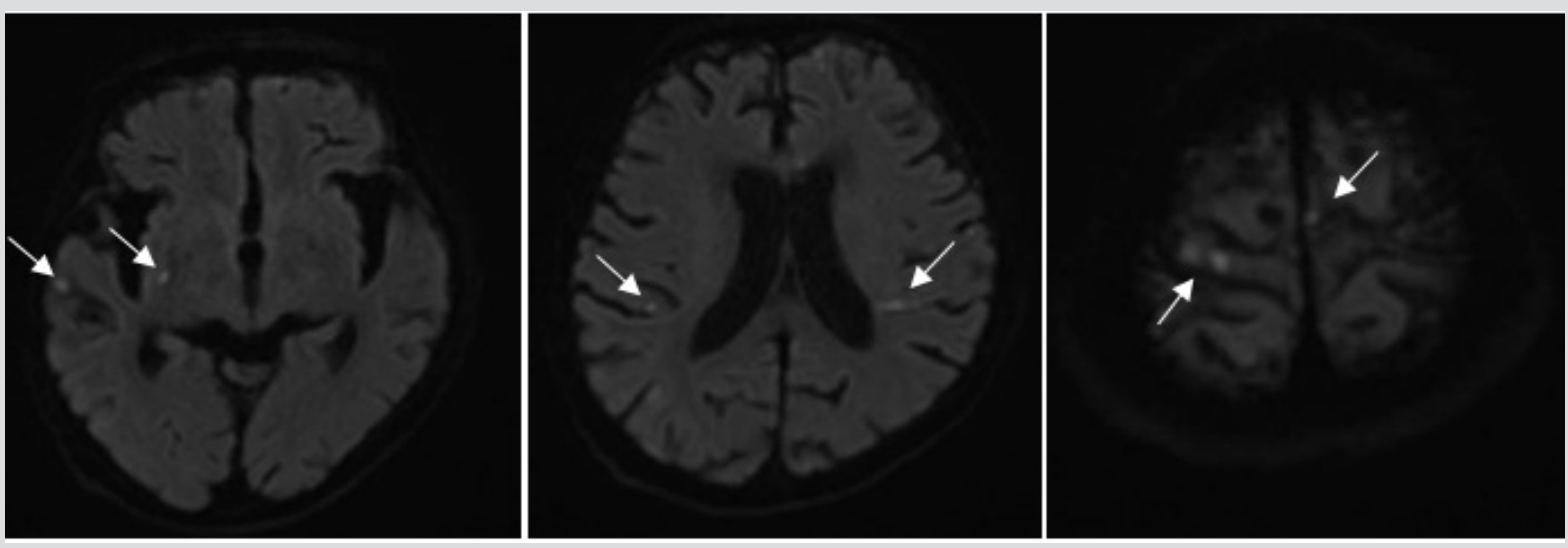

The patient was treated with meningeal administration of parenteral antibiotics and completed 4 weeks of antimicrobial therapy (comprising ceftriaxone, stepped down to benzylpenicillin), in view of possible infective endocarditis, with clinical improvement. No corticosteroids were started.

Interestingly, the patient had hypokalaemia and hypomagnesaemia on presentation, without vomiting or diarrhoea. Of note, serum calcium/ phosphate levels were normal on initial presentation. She only started developing hypophosphataemia on the 8th day of hospitalization. She continued to be hypokalaemic, hypomagnesaemic and hypophosphataemic despite aggressive initial replacement therapy, prompting further evaluation. Notably, the following urinary indices were raised: protein/creatinine ratio ( $1.53 \mathrm{mg} / \mathrm{dl}$ ), spot potassium/creatinine ratio (12.83 mEq/mmol), 24-hour urine potassium (58 mmol/day), fractional excretion of phosphate (22.89\%), fractional excretion of magnesium (14.37\%), 24-hour urine magnesium (9.07 mmol/day), fractional excretion of uric acid (13.91\%) and raised urine $\beta 2$-microglobulin/creatinine ratio $(3212 \mu \mathrm{g} / \mathrm{g})$. These biochemistry results were consistent with proximal tubular urinary losses, and gradually resolved during treatment of the patient's underlying infection (Table 1). However, no acidaemia or glycosuria was present. There were no hypotensive episodes during her hospitalization and her serum creatinine remained in the normal range with maintenance of good urine output throughout hospitalisation.

A Fanconi-like syndrome was hence suspected, presumably related to invasive pneumococcal infection, given lack of a secondary cause and its resolution following antimicrobial treatment. The patient's drug history and exposures were unremarkable. Blood and urinary screens for monoclonal bands were negative. She did not have any stigmata of rheumatological disease and both antinuclear antibody and extractable nuclear antigen were negative. Serum vitamin D levels were found to be low on day 14 of hospitalization and replacement therapy was initiated. This is unlikely a familial cause as the patient is elderly and did not subsequently require long-term oral electrolyte replacement. The patient's serum electrolytes had normalized by the time of her outpatient follow-up 1 week after antibiotic completion (Table 1). 


\begin{tabular}{|c|c|c|c|c|c|c|c|c|c|}
\hline Day & 1 & 6 & 10 & 12 & 13 & 14 & 21 & 35 & 47 \\
\hline Serum potassium (mmol/l) (3.5-5.1) & $2.8 \downarrow$ & $2.2 \downarrow$ & $2.9 \downarrow$ & 3.7 & $3.1 \downarrow$ & 3.5 & 3.5 & 4.2 & 3.9 \\
\hline Serum magnesium (mmol/l) (0.74-0.97) & $0.73 \downarrow$ & & 0.84 & 0.77 & $0.65 \downarrow$ & $0.64 \downarrow$ & $0.72 \downarrow$ & 0.82 & $0.71^{*} \downarrow$ \\
\hline Serum phosphate (mmol/l) (0.94- 1.50) & 1.11 & & & $0.91 \downarrow$ & $0.81 \downarrow$ & $0.89 \downarrow$ & 1.08 & 1.30 & 1.05 \\
\hline Serum parathyroid (intact) hormone (pmol/I) (1.60-6.90) & & & & & $8.65 \uparrow$ & & & & \\
\hline Serum 25 hydroxyvitamin D ( $\mu \mathrm{g} / \mathrm{I})(20-100)$ & & & & & & $6 \downarrow$ & & & 51 \\
\hline Serum uric acid (mmol/l) (143-330) & & & & & $122 \downarrow$ & & & & \\
\hline FE of uric acid (\%) & & & & & $13.91 \uparrow$ & & & & \\
\hline \multicolumn{10}{|l|}{ Urine $\beta 2$-microglobulin/creatinine ratio ( $\mu \mathrm{g} / \mathrm{g}$ ) } \\
\hline$(26-260)$ & & & & & $3212 \uparrow$ & & $712 \uparrow$ & & $617 \uparrow$ \\
\hline Urine protein/creatinine ratio (mg/dl) $(<0.2)$ & & $1.53 \uparrow$ & & & & & & & 0.19 \\
\hline Urine potassium/creatinine ratio (mEq/mmol) & & & $12.83 \uparrow$ & & & & $14.5 \uparrow$ & & \\
\hline 24-hour urine potassium (mmol/day) & & & & & 58 & & & & \\
\hline FE of magnesium (\%) & & & & $14.37 \uparrow$ & & & 3.88 & & 2.81 \\
\hline 24-hour urine magnesium (mmol/day) (1.97-4.94) & & & & $9.07 \uparrow$ & & & & & \\
\hline FE of phosphate (\%) & & & & $22.89 \uparrow$ & & & 10.56 & & 9.63 \\
\hline Tubular maximum phosphate reabsorption & & & & $0.71 \downarrow$ & & & 0.966 & & 1.03 \\
\hline \multicolumn{10}{|l|}{ 24-hour urine phosphate (mmol/day) } \\
\hline$(13.00-42.00)$ & & & & 26.04 & & & & & \\
\hline Urine $\mathrm{pH}(4.6-7.0)$ & & & & 7.0 & & & & & \\
\hline Urine glucose (dipstick) & & & & Negative & & & & & \\
\hline Progress and treatment history & $\begin{array}{l}\text { Days } \\
\text { IV ceftriaxone } \\
\text { IV azithromycin }\end{array}$ & $\longrightarrow$ & LP Clinic & $\underset{9}{9} \quad 10$ & $\begin{array}{c}\begin{array}{c}\text { Suspected } \\
\text { infe }\end{array} \\
\end{array}$ & $\begin{array}{r}\text { dose) } \\
\text { (herpes zost } \\
\text { PO levof }\end{array}$ & $\underset{\text { Started PO }}{\stackrel{\text { colecalciferol }}{\longrightarrow}}$ & $\begin{array}{c}28 \\
\\
\\
\end{array}$ & \\
\hline
\end{tabular}

Table 1. Serum, urinary results and antimicrobial therapy demonstrating progression and resolution of pneumococcal disease and Fanconi-like syndrome 
They remained normal without replacement therapy 7 weeks after her initial presentation of IPD. This further supports our suggestion that IPD occurred in association with this Fanconi-like syndrome, which resolved after treatment. Pre-existing idiopathic proximal tubulopathy is less likely in this case, given the previous normal serum electrolytes and evidence of complete resolution of tubulopathy after the acute infective episode.

\section{DISCUSSION}

Fanconi syndrome (FS) is caused by global dysfunction of the renal proximal tubule resulting in excessive urinary excretion of amino acids, glucose, phosphate, potassium, bicarbonate and uric acid ${ }^{[1]}$. This diagnosis should be considered when a patient presents with normal anion gap metabolic acidosis, hypokalaemia and urinary $\mathrm{pH}>5.3^{[2]}$. Acquired causes of FS in adults are shown in Table $2^{[1,3]}$.

\begin{tabular}{l} 
Drugs: \\
$\begin{array}{l}\text { Aminoglycosides } \quad \text { (gentamicin>tobramycin>amikacin), tetracyclines, nucleotide/nucleoside reverse } \\
\text { transcriptase inhibitors (adefovir, tenofovir, cidofovir, didanosine, stavudine), sodium valproate, carbonic } \\
\text { anhydrase inhibitors, azathioprine, chemotherapeutic agents (ifosfamide, cisplatin, carboplatin, imatinib), } \\
\quad \text { aspirin, deferasirox, fumaric acid, suramin }\end{array}$ \\
\hline $\begin{array}{l}\text { Toxic/metabolic causes: } \\
\text { Heavy metal poisoning (cadmium, lead, mercury, copper), glue sniffing, Chinese herbal remedies containing } \\
\quad \text { Aristolochia species, vitamin D deficiency }\end{array}$ \\
$\begin{array}{l}\text { Paraproteinaemias: } \\
\text { Sjogren's syndrome, light chain proteinuria, multiple myeloma, amyloidosis }\end{array}$ \\
\hline $\begin{array}{l}\text { Others: } \\
\text { Renal transplantation }\end{array}$
\end{tabular}

Drugs: anhydrase inhibitors, azathioprine, chemotherapeutic agents (ifosfamide, cisplatin, carboplatin, imatinib), aspirin, deferasirox, fumaric acid, suramin

Renal transplantation
Table 2. Causes of adult-onset Fanconi syndrome

Infective aetiologies are not thought to commonly cause proximal renal tubulopathy, although there have been reports of acquired FS in patients with Legionnaire's disease ${ }^{[4,5]}$. In these cases, the patients presented with hypophosphataemia, hypokalaemia, hypouricaemia and/ or hyponatraemia which resolved after the patients had recovered from Legionella pneumonia. Koda et al. reported elevated urinary $\beta 2-$ microglobulin in a patient, indicating severe renal tubular damage ${ }^{[5]}$. Kinoshita-Katahashi et al. speculated that direct infection by Legionella pneumophila in the proximal tubules disrupted mitochondrial function, resulting in $\mathrm{FS}^{[4]}$. Known to have a predilection for colonization in the proximal tubule, leptospirosis can also cause tubulointerstitial nephritis and proximal tubular dysfunction ${ }^{[6]}$. Tubular injury in these instances is believed to be mediated through Toll-like receptor-dependent pathways ${ }^{[6]}$.

While vitamin D deficiency may have played a contributing role in our patient, it is rarely implicated in causing proximal tubulopathy ${ }^{[2,7,8]}$. Our patient's initial serum calcium/phosphate levels were normal and her electrolytes started to normalize without continuous replacement, by the time of oral colecalciferol commencement. Although there have been no reports of IPD occurring in association with renal tubulopathy, we report a possible association with vitamin D deficiency, through exclusion of other causes. We postulate that direct infection by $S$. pneumoniae in the proximal tubules, resulting in tubular injury, could have occurred in our patient.

\section{CONCLUSION}

With this case, we highlight the interesting clinical phenomena of a patient with IPD who developed multiple electrolyte derangements likely secondary to Fanconi-like syndrome.

\section{REFERENCES}

1. Foreman JW. Fanconi syndrome. Pediatr Clin North Am 2019;66(1):159-167.

2. Ali SA, Tariq M. Successful treatment of proximal renal tubular acidosis and Fanconi syndrome with vitamin D replacement. Saudi J Kidney Dis Transpl 2016;27(4):812-815.

3. Hall AM, Bass P, Unwin RJ. Drug-induced renal Fanconi syndrome. QJM 2014;107(4):261-269.

4. Kinoshita-Katahashi N, Fukasawa H, Ishigaki S, Isobe S, Imokawa S, Fujigaki Y, et al. Acquired Fanconi syndrome in patients with Legionella pneumonia. BMC Nephrol 2013;14:171.

5. Koda R, Itoh R, Tsuchida M, Ohashi K, lino N, Takada T, et al. Legionella pneumonia complicated with acquired Fanconi syndrome. Intern Med 2018;57(20):2975-2980.

6. Yang CW. Leptospirosis renal disease: Understanding the initiation by Toll-like receptors. Kidney Int 2007;72(8):918-25. Doi: 10.1038/sj.ki.5002393

7. Taylor HC, Elbadawy EH. Renal tubular acidosis type 2 with Fanconi's syndrome, osteomalacia, osteoporosis, and secondary hyperaldosteronism in an adult consequent to vitamin D and calcium deficiency: effect of vitamin D and calcium citrate therapy. Endocr Pract 2006;12(5);559-67. doi: 10/4158/EP.12.5.559

8. Dalmark S, Erek E, Serdengecti K, Okar I, Ulku U, Basaran M. A case study of adult-onset hypophosphatemic osteomalacia with idiopathic Fanconi syndrome. Nephron 1996;72(1):121-2. doi: 10.1159/000188827 